\title{
Efeito da fumaça de cigarro e escovação simulada na cêramica vítrea de dissilicato de lítio CAD/CAM
}

\author{
Effect of cigarette smoke and simulated brushing on lithium disilicate vitreous CAD/CAM \\ Efecto del humo del cigarrillo y el cepillado simulado en CAD/CAM vítreo de disilicato de \\ litio
}

Magna Andréa Rabêlo Diógenes ${ }^{1}$, Francisca Tauliane Lemos de Castro ${ }^{1}$, Samara Kelly da Silva Cavalcante $^{1}$, Ana Carolina Matias Dinelly Pinto ${ }^{1}$, Pedro Henrique Chaves Isaias ${ }^{1}$, Natasha Muniz Fontes $^{1}$, Raul Anderson Domingues Alves da Silva ${ }^{1}$, Flávia Jucá Alencar e Silva ${ }^{1}$, Talita Arrais Daniel Mendes $^{1,2}$, Érika Matias Pinto Dinelly ${ }^{1 *}$.

\section{RESUMO}

Objetivo: Revisar acerca do efeito da fumaça do cigarro, associada ou não à escovação, na rugosidade superficial e na microdureza de uma cerâmica vítrea de dissilicato de lítio "Computer-Aided Design/ComputerAided Manufacturing" (CAD/CAM). Métodos: Pesquisaram-se os descritores cadastrados no MeSH "lithium disilicate", "smoking", "CAD CAM", "brushing", "rough", "hardness", combinados entre si pelo operador booleano "AND", na base de dados PubMed nos últimos 5 anos. Resultados: De acordo com a pesquisa, foram encontrados 625 artigos e selecionados 12 estudos in vitro. 5 estudos concluíram que as restaurações cerâmicas CAD/CAM apresentaram estabilidade de cor e de rugosidade da superfície, após escovação simulada e termociclagem. 4 estudos observaram que a escovação simulada e algumas bebidas aumentaram significativamente a rugosidade superficial da cerâmica de dissilicato de lítio. 3 estudos inferiram que a rugosidade superficial e a microdureza podem ser influenciadas pelos tratamentos de superfícies. Considerações finais: As cerâmicas de dissilicato de lítio CAD/CAM possuem propriedades satisfatórias em relação a sua resistência e estética, após exposição à fumaça do cigarro, associada ou não com a escovação.

Palavras-chave: Cerâmica, Dureza, Cigarro, CAD-CAM.

\begin{abstract}
Objective: Review the effect of cigarette smoke, associated or not with brushing, on the surface roughness and microhardness of a lithium disilicate glass ceramic "Computer-Aided Design/Computer-Aided Manufacturing" (CAD/CAM). Methods: Searched for the descriptors registered in MeSH "lithium disilicate", "smoking", "CAD CAM", "brushing", "rough", "hardness", combined among themselves by the Boolean operator "AND", in the database PubMed in the last 5 years. Results: According to the research, 625 articles were found and 12 in vitro studies were selected. 5 studies concluded that CAD/CAM ceramic restorations showed color stability and surface roughness after simulated brushing and thermocycling. 4 studies found that simulated brushing and some drinks significantly increased the surface roughness of lithium disilicate ceramic. 3 studies have inferred that surface roughness and microhardness can be influenced by surface treatments. Final considerations: $C A D / C A M$ lithium disilicate ceramics have satisfactory properties in terms of strength and aesthetics, after exposure to cigarette smoke, associated or not with brushing.
\end{abstract}

Key words: Ceramics, Hardness, Cigarette, CAD-CAM.

${ }^{1}$ Centro Universitário Católica de Quixadá (Unicatólica), Quixadá - CE. *E-mail: talita_arrais@hotmail.com

2 Universidade Federal do Ceará, Fortaleza - CE. 


\section{RESUMEN}

Objetivo: Revisar el efecto del humo del cigarrillo, asociado o no al cepillado, sobre la rugosidad superficial y la microdureza de una vitrocerámica de disilicato de litio "Diseño asistido por computadora/Fabricación asistida por computadora" (CAD/CAM). Métodos: Se buscaron los descriptores registrados en MeSH "disilicato de litio", "ahumado", "CAD CAM", "cepillado", "rugoso", "dureza", combinados por el operador booleano "Y", en la base de datos PubMed en los últimos 5 años. Resultados: De acuerdo con la investigación, se encontraron 625 artículos y se seleccionaron 12 estudios in vitro. Cinco estudios concluyeron que las restauraciones cerámicas CAD/CAM mostraron estabilidad de color y rugosidad de la superficie después de un cepillado y termociclado simulados. Cuatro estudios encontraron que el cepillado simulado y algunas bebidas aumentaron significativamente la rugosidad de la superficie de la cerámica de disilicato de litio. 3 estudios han inferido que la rugosidad superficial y la microdureza pueden verse influenciadas por los tratamientos superficiales. Consideraciones finales: Las cerámicas de disilicato de litio CAD/CAM tienen propiedades satisfactorias en términos de resistencia y estética, después de la exposición al humo del cigarrillo, asociado o no al cepillado.

Palabras clave: Cerámica, Dureza, Cigarrillo, CAD-CAM.

\section{INTRODUÇÃO}

A busca pela contante pela estética exerce influência em todos os âmbitos da vida do ser humano, resultando em uma exigência cada vez maior por um sorriso perfeito. A reposição de algum elemento dentário, bem como as mudanças em relação à cor, forma e tamanho dos dentes está diretamente relacionada ao bemestar do indivíduo (VICHI A, et al., 2018).

Atualmente, existem diversos materiais com a finalidade de reproduzir de forma fiel a estrutura dentária, dentre as quais, podem-se citar as cerâmicas odontológicas como as feldspáticas, leucitas e dissilicato de lítio, que são cerâmicas bastante utilizadas em reabilitações estéticas por apresentarem uma matriz vítrea em sua composição, o que proporciona estética e translucidez as peças protéticas, já as cerâmicas a base de alumína e zircônia, são mais utilizadas em reabilitações que requerem resistência. Dentre as cerâmicas citadas, o dissilicato de lítio se destaca por apresentar propriedades como aumento da resistência sem comprometimento estético, além de apresentar excelente resistência flexural, o que proporciona uma maior versatilidade de indicações (VICHI A, et al., 2018).

Diferentes técnicas de processamento das cerâmicas odontológicas podem ser utilizadas, como o método de estratificação, fundição por suspensão, prensagem e fresagem ou usinagem. Destaca-se este último método, o qual pode utilizar a tecnologia CAD-CAM para a sua execução. O sistema CAD/CAM é uma forma de processamento para blocos cerâmicos na qual a sua programação e técnicas ocorrem no computador. Com o auxílio de um scanner as informações são obtidas em um modelo de gesso ou na cavidade oral, após, há uma conexão ao computador em que será planejado o protocolo clínico que será seguido. Posteriormente, a restauração é fresada (FLURY S, et al., 2017).

Trata-se de um sistema rápido e preciso quando comparado aos outros métodos, o qual busca uma maior semelhança entre a coroa confeccionada e o dente natural. Esse sistema é eficaz, sendo indicado para cerâmicas livres de metais, sendo mais eficaz quando usado na região anterior em relação à região posterior, mas vale salientar que isso varia de acordo com o material e a marca comercial (MOTA GE, et al., 2017; YIN $\mathrm{R}$, et al., 2019). A simplicidade da técnica e a velocidade com que essas restaurações são realizadas representam um benefício, porém o custo elevado é uma desvantagem (MENEZES SM, et al., 2015).

As cerâmicas odontológicas, em especial, as cerâmicas de dissilicato de lítio, possuem excelentes propriedades em relação à estética e à durabilidade. Sabe-se que alguns fatores extrínsecos, tais como o cigarro e os alimentos, podem alterar as propriedades das cerâmicas odontológicas, resultando em menor durabilidade das próteses. Levando em consideração que a quantidade de fumantes é significativa em várias regiões do mundo, verificar se a fumaça do cigarro irá interferir em propriedades superficiais de cerâmicas, 
como microdureza e rugosidade superficial e, consequentemente, na sua durabilidade, resistência e estética é fundamental para os avanços da odontologia restauradora (MENEZES SM, et al., 2015).

Dessa forma, o objetivo deste trabalho é revisar acerca do efeito da fumaça do cigarro, associada ou não à escovação, na rugosidade superficial e na microdureza de uma cêramica vítrea de dissilicato de litio $\mathrm{CAD} / \mathrm{CAM}$.

\section{MÉTODOS}

Realizou-se uma revisão de literatura de natureza descritiva, através da análise de estudos que abordassem o efeito da fumaça do cigarro e da escovação na rugosidade superficial e microdureza na cerâmica de dissilicato de lítio. $O$ desenvolvimento deste trabalho foi realizado através da análise de artigos científicos obtidos a partir das bases de dados Sistema Online de Busca e Análise de Literatura Médica (PubMed). Os descritores usados na busca, cadastrados na MeSH (Medical Subject Headings) foram: lithium disilicate, smoking, CAD CAM, brushing, rough, hardness, sendo "AND" o operador Booleano. A seleção dos artigos foi feita através de títulos e resumos como objetivo de um apanhado geral sobre o tema. Foram incluídos artigos da língua inglesa e delimitado no período de 2016 a 2021. Foram excluídos estudos não encontrados na íntegra, indisponíveis e trabalho em outros idiomas e com estudos inconclusivos. Após a leitura de títulos e resumos, foram selecionados 12 artigos (Figura 1).

Figura 1- Fluxograma metodológico.

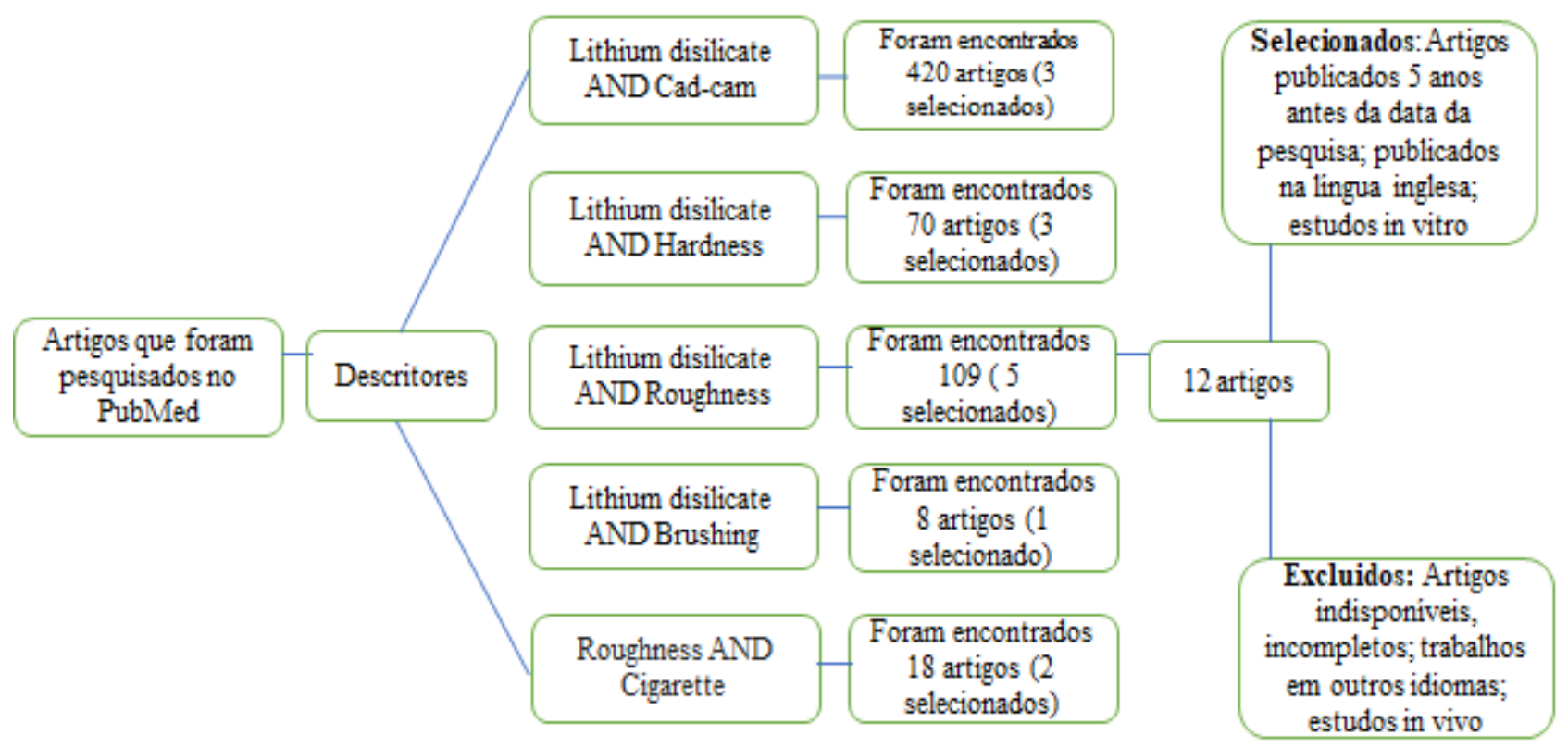

Fonte: Diógenes MAR, et al., 2021.

\section{RESULTADOS}

Através da pesquisa realizada na base de dados PubMed utilizando os descritores: dissilicato de lítio, CAD/CAM, rugosidade, escovação, dureza e cigarro. Foram encontrados 625 artigos e selecionados 12. Os artigos foram selecionados após a leitura de titulos e resumos (Quadro 1). 
Quadro 1 - Descrição dos artigos selecionados: autor/ano, título, metodologia e resultados.

\begin{tabular}{|c|c|c|c|}
\hline Autor/Ano & Título & Metodologia & Resultados \\
\hline $\begin{array}{l}\text { GARZA LA, et } \\
\text { al., } 2016\end{array}$ & $\begin{array}{c}\text { Effect of toothbrushing on shade } \\
\text { and surface roughness of } \\
\text { extrinsically stained pressable } \\
\text { ceramics }\end{array}$ & $\begin{array}{l}\text { Dois materiais, à base de leucita (IPS Empress Esthetic [EE]; Ivoclar } \\
\text { Vivadent AG) e cerâmica à base de dissilicato de lítio (IPS e.max } \\
\text { Press [EP]; Ivoclar Vivadent AG), foram estudados. Para cada } \\
\text { material, foram confeccionados } 24 \text { corpos-de-prova em forma de } \\
\text { disco, } 10 \mathrm{~mm} \text { (diâmetro) x } 3 \mathrm{~mm} \text { (altura). Três métodos diferentes (n = } \\
\text { 8) de aplicação de corantes extrínsecos foram realizados em cada } \\
\text { material: apenas esmaltado (G, grupo controle); manchado e depois } \\
\text { glaceado (SG); e manchados e vitrificados juntos (T). }\end{array}$ & $\begin{array}{l}\text { IPS Empress sem mudanças } \\
\text { após } 12 \text { anos de escovação } \\
\text { simulada IPS e.max obteve } \\
\text { mudanças na tonalidade e } \\
\text { rugosidade após } 12 \text { anos de } \\
\text { escovação simulada. }\end{array}$ \\
\hline $\begin{array}{l}\text { LAWSON NC, } \\
\text { BURGESS JO, } \\
2016\end{array}$ & $\begin{array}{c}\text { Gloss and Stain Resistance of } \\
\text { Ceramic-Polymer CAD/CAM } \\
\text { Restorative Blocks }\end{array}$ & $\begin{array}{l}\text { Os espécimes (4 mm) foram seccionados de: Enamic (cerâmica } \\
\text { infundida com polímero), LAVA Ultimate (polímero reforçado com } \\
\text { nanocerâmica), e.max (dissilicato de lítio), Paradigma } C \text { (porcelana) e } \\
\text { Paradigma MZ100 (compósito). Os espécimes foram polidos a úmido } \\
\text { em uma roda de polimento para papel de silício de granulação } 320 \\
\text { (não polido, } \mathrm{N}=8 \text { ) ou papéis de carboneto de silício de granulação } \\
2.000 \text { seguidos por uma pasta de alumina de } 0,05 \mu \mathrm{m} \text { (polido, } \mathrm{N}=8 \text { ). }\end{array}$ & $\begin{array}{l}\text { Dissilicato de lítio e zircônia } \\
\text { apresentaram mais forte, duro do } \\
\text { que os outros que apresentaram } \\
\text { sinais abrasivos em esmalte. } \\
\text { Resina composta sinais de } \\
\text { fadiga. Polímero de cerâmica } \\
\text { mostrou abrasividade em } \\
\text { esmalte. }\end{array}$ \\
\hline $\begin{array}{l}\text { EL GAMAL A, et } \\
\text { al., } 2017\end{array}$ & $\begin{array}{c}\text { Microhardness evaluations of } \\
C A D / C A M \text { ceramics irradiated } \\
\text { with } C O_{2} \text { or Nd:YAP laser }\end{array}$ & $\begin{array}{l}\text { Sessenta discos de cerâmica CAD / CAM foram preparados e } \\
\text { agrupados por material, ou seja, cerâmica de dissilicato de lítio (Emax } \\
\text { CAD) e cerâmica de zircônia (Emax ZirCAD). A irradiação de laser na } \\
\text { superfície do material foi realizada com um laser de dióxido de } \\
\text { carbono a } 5 \text { Watt }(\mathrm{W}) \text { ou } 10 \mathrm{~W} \text { de potência em modo contínuo (modo } \\
\mathrm{CW} \text { ), ou com um laser de neodímio: ítrio perovskita de alumínio (Nd: } \\
\text { YAP) a } 10 \mathrm{~W} \text { em grafite e superfícies não grafite. A dureza Vickers foi } \\
\text { testada em } 0,3 \mathrm{~kg} f \text { para dissilicato de lítio e } 1 \mathrm{~kg} f \text { para zircônia. }\end{array}$ & $\begin{array}{l}\text { Emax CAD irradiados com } \\
\text { CO } 2 \text { aumentou microdureza. } \\
\text { Emax Zir CAD irradiada com Nd: } \\
\text { YAP diminuiu microdureza. }\end{array}$ \\
\hline $\begin{array}{l}\text { MOTA GE, et } \\
\text { al., } 2017\end{array}$ & $\begin{array}{c}\text { The effect of milling and } \\
\text { postmilling procedures on the } \\
\text { surface roughness of CAD/CAM } \\
\text { materials }\end{array}$ & $\begin{array}{l}\text { Blocos de cerâmica Mark II, IPS Empress CAD, IPS e.max CAD, } \\
\text { Suprinity e Enamic, e blocos de resina composta Lava Ultimate foram } \\
\text { moídos no CEREC MCXL. Foram obtidas dez amostras planas de } \\
\text { cada material. O teste de rugosidade superficial (Ra) foi realizado } \\
\text { antes e após a moagem, cristalização, polimento e glaze quando } \\
\text { indicado, seguido de análises em MEV e AFM. }\end{array}$ & $\begin{array}{c}\text { A rugosidade superficial } \\
\text { aumentou em todos os materiais } \\
\text { na etapa de fresagem. O } \\
\text { dissilicato de lítio se apresentou } \\
\text { mais rugosa que outros materiais. }\end{array}$ \\
\hline
\end{tabular}




\begin{tabular}{|c|c|c|c|}
\hline Autor/Ano & Título & Metodologia & Resultados \\
\hline $\begin{array}{l}\text { YUAN JCC, et } \\
\text { al., } 2017\end{array}$ & $\begin{array}{l}\text { Effect of brushing and } \\
\text { thermocycling on the shade and } \\
\text { surface roughness of CAD-CAM } \\
\text { ceramic restorations }\end{array}$ & $\begin{array}{l}\text { A cerâmica CAD de dissilicato de lítio }(n=90) \text { e a cerâmica de zircônia } \\
(n=90) \text { foram estudadas. Todas as amostras foram cristalizadas / } \\
\text { sinterizadas, caracterizadas e envidraçadas de acordo com as } \\
\text { recomendações do fabricante. Os espécimes foram divididos em } 9 \\
\text { grupos diferentes: B, TC e uma combinação de B mais TC }(B+T C) \text {. A } \\
\text { escovação foi realizada em } 50.000,100.000 \text { e } 150.000 \text { ciclos, } \\
\text { simulando um ambiente bucal de } 5,10 \text { e } 15 \text { anos. }\end{array}$ & $\begin{array}{l}\text { Na cerâmica de dissilicato de lítio: } \\
\text { sem mudanças. Em zircônia } \\
\text { houve uma maior diferença de cor } \\
\text { em } 15 \text { anos comparado com } 5 \\
\text { anos. Após escovação simulada, } \\
\text { houve aumento da rugosidade }\end{array}$ \\
\hline $\begin{array}{c}\text { VICHI A, et al., } \\
2018\end{array}$ & $\begin{array}{l}\text { Effect of Finishing and Polishing } \\
\text { on Roughness and Gloss of } \\
\text { Lithium Disilicate and Lithium } \\
\text { Silicate Zirconia Reinforced } \\
\text { Glass Ceramic for CAD/CAM } \\
\text { Systems }\end{array}$ & $\begin{array}{l}\text { Um total de } 24 \text { blocos de Suprinity e } 24 \text { de e.max foram cortados em } \\
\text { forma de cunha usando uma unidade de moagem InLab MC-XL. Após } \\
\text { a cristalização, as } 24 \text { cunhas Suprinity foram divididas em quatro } \\
\text { subgrupos: grupo A.1: Suprinity Polishing Set Clinical usado por } 30 \\
\text { segundos e grupo A.2: por } 60 \text { segundos; grupo A.3: Pasta VITA } \\
\text { Akzent Plus; e grupo A.4: spray. As cunhas de } 24 \text { e.max (grupo B) } \\
\text { foram divididas em quatro subgrupos de acordo com o procedimento } \\
\text { de acabamento: grupo B.1: Optrafine Ceramic Polishing System por } 30 \\
\text { segundos e grupo B.2: por } 60 \text { segundos; grupo B.3: pasta IPS e.max } \\
\text { CAD Crystall / Glaze; e grupo B.4: spray. }\end{array}$ & $\begin{array}{l}\text { Suprinity apresentou menor } \\
\text { rugosidade do que a E.max. } \\
\text { Suprinity apresentou brilho maior } \\
\text { que E.max }\end{array}$ \\
\hline $\begin{array}{l}\text { OZDOGAN A, } \\
\text { DUYMUS Z, } \\
2018\end{array}$ & $\begin{array}{l}\text { Investigating the Effect of } \\
\text { Different Surface Treatments on } \\
\text { Vickers Hardness and Flexural } \\
\text { Strength of Zirconium and } \\
\text { Lithium Disilicate Ceramics }\end{array}$ & $\begin{array}{l}\text { Foram utilizados } 50 \text { espécimes de cerâmica de zircônia e } 50 \text { dissilicato } \\
\text { de lítio. Após identificar um grupo como controle, a retificação, jato de } \\
\text { areia, CoJet e laser Er: YAG foram aplicados na superfície das } \\
\text { amostras }(n=10) .\end{array}$ & $\begin{array}{l}\text { Diferenças significativas no } \\
\text { tratamento de superfície em } \\
\text { dureza Vickers em zircônia e } \\
\text { dissilicato de lítio, mais sem } \\
\text { diferenças em relação à } \\
\text { resistência flexural. }\end{array}$ \\
\hline $\begin{array}{l}\text { STRASSER T, } \\
\text { et al., } 2018\end{array}$ & $\begin{array}{l}\text { Roughness, surface energy, and } \\
\text { superficial damages of } \\
\text { CAD/CAM materials after } \\
\text { surface treatment }\end{array}$ & $\begin{array}{c}\text { Amostras foram feitas de dez materiais CAD / CAM (Celtra Duo, } \\
\text { Degudent, D; Vita Suprinity, Vita, D; E.max CAD, Ivoclar-Vivadent, FL; } \\
\text { E.max ZirCAD, Ivoclar-Vivadent, FL; Vita Enamic, Vita, D; Cerasmart, } \\
\text { GC, B; LAVA Ultimate, 3M, D; SHOFU Block HC, SHOFU, US; } \\
\text { Grandio Blocs, VOCO, D; BRILLIANT Crios, Coltene, CH) e pré- } \\
\text { tratado para representar procedimentos clínicos (Hf } 20 \text { s / 5\%; ácido } \\
\text { fosfórico } 20 \mathrm{~s} / 37 \%\end{array}$ & $\begin{array}{l}\text { A análise SEM mostrou danos } \\
\text { após o pré-tratamento, } \\
\text { dependendo do material. }\end{array}$ \\
\hline
\end{tabular}




\begin{tabular}{|c|c|c|c|}
\hline Autor/Ano & Título & Metodologia & Resultados \\
\hline $\begin{array}{l}\text { ALENCAR- } \\
\text { SILVA FJ, et al., } \\
2019\end{array}$ & $\begin{array}{l}\text { Effect of beverage solutions and } \\
\text { toothbrushing on the surface } \\
\text { roughness, microhardness, and } \\
\text { color stainability of a vitreous } \\
\text { CAD-CAM lithium disilicate } \\
\text { ceramic }\end{array}$ & $\begin{array}{l}\text { Corpos de prova }(\mathrm{N}=160) \text { foram divididos em } 2 \text { grupos de acordo } \\
\text { com o método de tratamento de superfície: polimento com discos } \\
\text { abrasivos ou aplicação de glaze. Cada grupo foi subdividido }(n=8) \text { de } \\
\text { acordo com a solução de imersão e escovação: água destilada e água } \\
\text { destilada mais escovação; café e café com escovagem; chá preto e } \\
\text { chá preto com escovagem; vinho tinto e vinho tinto com escovação; e } \\
\text { coca-cola mais escovação. }\end{array}$ & $\begin{array}{l}\text { Independente da solução, a } \\
\text { rugosidade se mostrou } \\
\text { aumentada, a diminuição da } \\
\text { dureza teve por influência as } \\
\text { soluções e tratamento de } \\
\text { superfície e independente do } \\
\text { tratamento a cor teve por } \\
\text { influencia as soluções. }\end{array}$ \\
\hline $\begin{array}{l}\text { MACIEL CL, et } \\
\text { al., } 2019\end{array}$ & $\begin{array}{l}\text { Influence of polishing systems } \\
\text { on roughness and color change } \\
\text { of two dental ceramics }\end{array}$ & $\begin{array}{c}\text { Foram fabricados } 88 \text { discos de cerâmicas, } 44 \text { cerâmicas de } \\
\text { estratificação de nano-fluorapatita (IPS e.max Ceram. Grupo C) e } 44 \\
\text { discos de cerâmica de dissilicato de lítio prensada (IPS e. Max Press - } \\
\text { Grupo P). Cada grupo foi dividido em } 4 \text { subgrupos de acordo com os } \\
\text { tratamentos de superfície: (G) Glaze, (S) Sistema de polimento Shofu } \\
\text { (Shofu Inc.), (E) Sistema de polimento Edenta AG, (KG) ponta de } \\
\text { granulação de diamante de } 30 \mu \text { m. Foram realizadas medições de } \\
\text { rugosidade superficial (RS) e mudançá de cor }(\Delta E) \text { após os } \\
\text { tratamentos superficiais, antes e } 12 \text { dias após a imersão na solução de } \\
\text { café. }\end{array}$ & $\begin{array}{l}\text { Valores de Rugosidade superficial } \\
\text { aceitável clinicamente. Existe } \\
\text { uma forte correlação entre a } \\
\text { rugosidade da superfície e a } \\
\text { mudança de cor de cerâmicas } \\
\text { testadas. }\end{array}$ \\
\hline $\begin{array}{l}\text { SUDRÉ JP, et } \\
\text { al., } 2020\end{array}$ & $\begin{array}{l}\text { Influence of Surface Treatment } \\
\text { of Lithium Disilicate on } \\
\text { Roughness and Bond Strength }\end{array}$ & $\begin{array}{l}\text { Um total de } 100 \text { discos de cerâmica IPS e.max Press reforçado com } \\
\text { dissilicato de lítio foram preparados da seguinte forma: o grupo } 1 \\
\text { permaneceu sem tratamento (grupo controle); nos grupos } 5-20,5-40 \text { e } \\
\text { 5-60, as superfícies foram atacadas com HF 5\% por } 20,40 \text { e } 60 \\
\text { segundos, respectivamente; nos grupos } 10-20,10-40 \text { e } 10-60 \text {, as } \\
\text { superfícies foram condicionadas com HF } 10 \% \text { por } 20,40 \text { e } 60 \\
\text { segundos, respectivamente; e nos grupos } 10-20 \mathrm{P}, 10-40 \mathrm{P} \text { e } 10-60 \mathrm{P} \text {, } \\
\text { as superfícies foram condicionadas com HF } 10 \% \text { por } 20,40 \text { e } 60 \\
\text { segundos, respectivamente, seguido de tratamento com ácido } \\
\text { fosfórico } 37 \% \text { por } 5 \text { segundos. }\end{array}$ & $\begin{array}{l}\text { A rugosidade teve interferências } \\
\text { da concentração e tempo de } \\
\text { exposição ao ácido. O HF a } 10 \% \\
\text { por } 40 \text { segundos obteve maior } \\
\text { rugosidade, já a ligação foi } \\
\text { afetada pelo tempo e exposição } \\
\text { ao ácido. }\end{array}$ \\
\hline $\begin{array}{c}\text { SCHESTATSKY } \\
\text { R, et al., } 2020\end{array}$ & $\begin{array}{l}\text { Microstructure, topography, } \\
\text { surface roughness, fractal } \\
\text { dimension, internal and marginal } \\
\text { adaptation of pressed and milled } \\
\text { lithium-disilicate monolithic } \\
\text { restorations }\end{array}$ & $\begin{array}{c}\text { Cem preparações idênticas para coroas monolíticas foram } \\
\text { confeccionadas com material análogo à dentina (resina epóxi } \\
\text { G10). Uma das preparações foi digitalizada e uma coroa monolítica } \\
\text { em dissilicato de lítio foi planejada no sistema CAD. Cinquenta coroas } \\
\text { foram fresadas em cera e submetidas à prensagem (IPS e.max } \\
\text { Press), enquanto } 50 \text { coroas foram usinadas em CAD / CAM (IPS } \\
\text { e.max CAD) e posteriormente cristalizadas. }\end{array}$ & $\begin{array}{l}\text { Na técnica } C A D / C A M \text { as } \\
\text { características topográficas } \\
\text { ficaram mais suaves, maior } \\
\text { dimensão fractal, mais } \\
\text { homogêneas em comparação a } \\
\text { técnica de prensagem. }\end{array}$ \\
\hline
\end{tabular}

Fonte: Diógenes MAR, et al., 2021. 


\section{DISCUSSÃO}

As cerâmicas odontológicas são materiais com aspecto semelhante aos dentes naturais, apresentando transparência, fluorescência, opacidade, boas propriedades ópticas, alta resistência, rigidez, estabilidade, além de serem biocompatíveis. Dentre as cerâmicas disponíveis para o sistema CAD/CAM, o dissilicato de lítio apresenta excelentes propriedades em relação à resistência e estética. Estas cerâmicas apresentam versatilidade, como alta resistência flexural, boas propriedades estéticas e grau de desgaste semelhante ao da estrutura dental, apresentando diferentes formas de cimentação (AL HAMAD KQ, et al., 2018).

A cerâmica de dissilicato de lítio é indicada para laminados cerâmicos, inlays, onlays, coroas unitárias anteriores e posteriores, próteses parciais fixas anteriores e posteriores, e prótese adesiva anterior. No que se refere à adaptação marginal, embora a zircônia seja um material com boa adaptação, alguns estudos demonstram que o dissilicato de lítio apresenta uma melhor resistência (ANADIOTI E, et al., 2015; ZELTNER M, et al., 2017).

A escolha do material restaurador é de grande relevância para a longevidade da prótese. Sabendo-se da grande procura pela estética, a estabilidade de cor é algo a ser priorizado, que se deve à espessura da cerâmica e à rugosidade superficial. A cerâmica pode estar sujeita a pigmentação, alteração na translucidez, brilho e rugosidade. Fatores como a aplicação, a fabricação e a queima estão diretamente relacionados com o aspecto da porcelana. A decomposição da cerâmica pode ser influenciada por altas temperaturas, acúmulo de biofilme e bebidas ácidas, como o refrigerante e o café (DOS SANTOS DM, et al., 2017).

Tendo em vista esses fatores e relacionando com os estudos incluídos para síntese qualitativa nessa revisão, Lawson NC e Burgess JO (2016) analisaram as propriedades mecânicas da zircônia, dissilicato de lítio, três tipos de resina composta e um polímero infiltrado por cerâmica, no sistema CAD/CAM. De acordo com os resultados, o dissilicato de lítio, a zircônia e o polímero reforçado por cerâmica em esmalte apresentaram sinais de abrasividade, já as resinas compostas mostraram-se com sinais de fragilidade. Entre essas, a que se apresentou com maior dureza foi o dissilicato de lítio e a zircônia. Com isso, verifica-se que as resinas compostas e polímeros infiltrados necessitam de espessura semelhante ao dissilicato de lítio mesmo com resistência ao desgaste adequada.

Em outro estudo, Schestatskya R, et al. (2020) avaliaram a topografia, a microestrutura, a rugosidade, a adaptação interna e marginal em diferentes formas de apresentação da cerâmica a base de dissilicato de lítio, prensada e frezada. A técnica CAD/CAM evidenciou uma topografia e rugosidade suave, mais homogênea $e$ maior adaptação marginal quando comparado com a prensagem.

No que diz respeito à rugosidade, foi constatada uma superfície mais heterogênea e áspera na técnica de prensagem, ademais, essa técnica demostrou uma maior adaptação das coroas na margem. Embora a técnica CAD/CAM e a prensagem apresentem composições similares, a técnica de prensagem do dissilicato de lítio é mais propensa a alterações em sua degradação estrutural quando comparado com o dissilicato de lítio fresado, visto que a o bloco fresado é confeccionado de forma sintetizada, compactada, com maior resistência mecânica e menos propensa a falhas em seu interior (SCHESTATSKYA R, et al., 2020).

A princípio, Strasser T, et al. (2018) examinaram o tratamento de superficie CAD/CAM em materiais como cerâmicas vítreas, zircônia, cerâmica infiltrada por resina e compósitos a base de resina. Foi constatado que esses materiais precisam de um pré-tratamento individual para ter uma proteção superficial. Os tratamentos realizados foram o jateamento de baixa pressão para zircônia; ácido fluorídrico para cerâmicas vítreas; ácido fluorídrico de erosão ou jateamento em cerâmica infiltrado com resina e os compósitos com jateamento e baixa pressão. $O$ tratamento aumentou a rugosidade superficial e a energia de superfície com danos aceitáveis, certificando que o pré-tratamento irá causar uma superfície aprimorada.

Posteriormente, Sudré JP, et al. (2019) buscaram avaliar o efeito de diferentes concentrações de ácido fluorídrico sobre rugosidade superficial e a força de ligação entre a cimento e a resina de cerâmica autoadesiva. Denotaram que a rugosidade superficial foi influenciada pela concentração e o tempo de exposto ao ácido. A aplicação de $10 \%$ de ácido fluorídrico por 40 segundos obteve maior rugosidade, contudo, não houve diferença significativa em relação à força de ligação. No que diz respeito à força de ligação, foi afetado 
somente pelo tempo de exposição. O condicionamento da cerâmica pode alterar a estrutura superficial, logo, afetar a força de ligação. É necessário o tratamento da superfície para ocorrer o microcisalhamento da força de ligação da cerâmica e o autoadesivo.

Através de um estudo in vitro, Mahross ZH, et al. (2015) analisaram o efeito da fumaça de cigarro na rugosidade superficial em dois tipos de dentaduras: uma resina polimerizada a calor e outra polimerizada a luz. As amostras que foram expostas a fumaça de cigarro apresentaram mudanças significativas na rugosidade superficial. O grupo II, representado pelas resinas acrílicas expostas ao tabagismo, apresentou tais mudanças, porém o grupo IV, representado pelas resinas polimerizadas expostas ao tabagismo não apressentou qualquer alteração. O grupo I e o II, representados pelas resinas polimerizadas de calor e luz, respectivamente, não foram expostas a fumaça de cigarro e não houve alteração na rugosidade.

O cigarro tem várias substâncias tóxicas, quando a fumaça entra em contato com a superfície dental há um comprometimento estético. O fumo do tabaco reduz a luminosidade, além de tornar a cerâmica amarelada, e as superfícies ásperas apresentaram maior probabilidade de pigmentação. A pigmentação nesses materiais pode ser por conta do alcatrão presente no fumo do tabaco, ressalta-se ainda que mudanças na temperatura alteram o manchamento das cerâmicas (MAHORSS ZH, et al., 2015).

Segundo Ayaz EA, et al. (2014) a fumaça do cigarro altera a rugosidade e a estabilidade de cor de dentes artificiais, enquanto os dentes de porcelana apresentam-se mais estáveis que os dentes de resina acrílica. Em um estudo, Alandia-Roman CC, et al. (2013) analisaram o efeito da fumaça do cigarro sobre a estabilidade de cor e a rugosidade superficial, observando-se alteração de cor, porém, isso pode ocorrer por conta da composição química do material, alimentação e deposição de corantes.

Ademais, esses autores verificaram que a falta de polimento em cerâmicas que são submetidas à fumaça do cigarro aumenta a rugosidade superficial. Além da fumaça do cigarro interferir na estabilidade de cor e aspereza da superfície, o hábito de fumar está relacionado com o acúmulo de microrganismos, tais como Streptococcus mutans e Cândida albicans, e estes por sua vez interferem na adesão da cerâmica com a estrutura dentária. Dessa forma, o fumo mostrou que os Streptococcus apresentaram maior adesão na película adquirida enquanto o $C$. Albicans mostrou maior adesão em resina acrílica (MAHORSS ZH, et al., 2015).

Por vez, Theobaldo JD, et al. (2016) avaliaram o efeito da fumaça de cigarro na resistência de união das resinas compostas em relação à dentina. A mesma apresentou uma menor união para os grupos expostos a fumaça do cigarro, ou seja, essa exposição prejudicou a adesão da dentina com os adesivos. No esmalte não houve diferença de união nos grupos com e sem exposição da fumaça de cigarro, a diferença relatada foi em relação entre os adesivos.

Além disso, Mota GE, et al. (2018) analisaram a rugosidade superficial de materiais no momento da fresagem e pós-fresagem em cinco cerâmicas e uma resina composta. Foi observado um aumento na rugosidade da superficie na etapa da fresagem para todos os materiais, porém, há um aumento na rugosidade em materiais parcialmente cristalizados (IPS e.max ${ }^{\circledR}$ CAD e Suprinity). Na etapa de pós-fresagem 0 polimento reduziu a rugosidade de materiais cristalizados (Mark II, IPS Empress $\AA^{\circ}$ CAD, Enamic $\circledast$, e Lava Ultimate $\left.{ }^{\circledR}\right)$, os que são parcialmente cristalizados necessitou de vitrificação.

Semelhantemente, Vichi A, et al. (2018) avaliaram a efetividade do acabamento e polimento sobre o brilho e a rugosidade superficial de cerâmicas como VITA Suprinity ${ }^{\circledR}$ e IPS e.max ${ }^{\circledR}$ CAD. Com isso, verificou que em relação a rugosidade superficial a Suprinity apresentou menor rugosidade comparado com o IPS e Max CAD. O brilho se mostrou superior na cerâmica Suprinity. Cerâmicas a base de sílica CAD/CAM necessitam de um acabamento manual e polimento durante sessenta segundos com pasta de vidro para reduzir a rugosidade, e o mesmo tempo deve ser usado para obter um brilho superior.

Desse modo, Maciel CL, et al. (2019) averiguaram o efeito do polimento na rugosidade superficial e a cor da cerâmica após a imersão no café. Observaram que as cerâmicas, IPS. e.max, cerâmica de estratificação e IPS e.max prensada, quando são imersas por 12 dias na solução de café sofrem alteração de cor, não 
importando qual seja o tratamento da superfície, e quanto maior a rugosidade superficial maior a alteração de cor. O polimento apresentou bom desempenho e a cor foi considerada aceitável.

Em um estudo, a Coca-Cola e o suco de laranja foram armazenados em temperatura ambiente e o chá, café e água foram acondicionados em uma temperatura de $50^{\circ}$, observando-se que o café foi o que mais afetou a estabilidade de cor das porcelanas. Em relação à rugosidade superficial, o suco de laranja gerou maior alteração em decorrência da sua acidez (JAIN C, et al., 2013).

Em concordância com esse estudo, Daryakenari G, et al. (2018) avaliaram a capacidade de desgaste e rugosidade de quatro cerâmicas diferentes, Enamic, Suprinity, Vita Mark II e E-max. Antes e após o desgaste, a Vita Mark II apresentou maior rugosidade. Quando as porcelanas foram submetidas ao desgaste, observouse a redução da rugosidade nos grupos. As espécies que apresentaram menor desgaste em esmalte foram os grupos Emanic e Suprinity.

Com o intuito de reduzir a rugosidade na superfície é necessário realizar polimento, para que a superfície fique lisa o suficiente para refletir maior quantidade de luz. É importante ressaltar que a cor da cerâmica pode ser afetada, pois quanto maior a rugosidade maior a irregularidade e consequentemente a propagação de luz. Dessa forma, o glazeamento demonstrou que quanto mais polida for a superfície, menor será a instabilidade de cor DARYAKENARI G, et al., 2018).

Através de um estudo in vitro, El Gamal A, et al. (2017) avaliaram a microdureza de uma cerâmica $\mathrm{CAD} / \mathrm{CAM}$ antes e depois de um tratamento térmico com laser $\mathrm{Nd}$ : YAP e $\mathrm{CO}^{2}$, sendo observado que a cerâmica irradiada com $\mathrm{CO}^{2}$ teve um aumento na microdureza, representado pela cerâmica de dissilicato de lítio (Emax CAD) e pelo dióxido de zircônia (Emax ZirCAD). Já a cerâmica Emax ZirCAD irradiada com o laser, diminui a dureza. Na Emax CAD a temperatura não influenciou os valores de microdureza, diferentemente da Emax ZirCAD, com isso o laser $\mathrm{CO}^{2}$ aumenta a microdureza da cerâmica de dissilicato de lítio.

Ademais, Ozdogan A e Duymus DY (2018) averiguaram sobre zircônia e o dissilicato de lítio o efeito de diversos tratamentos de superfície de resistência flexural e dureza Vickers. Os tratamentos de superfície aumentaram a dureza Vickers das cerâmicas, porém, não afetaram a resistência flexural.

Em outro estudo, Garza LA, et al. (2016) verificaram o efeito da escovação na tonalidade e rugosidade superficial de cerâmicas extrinsecamente coradas. Para a cerâmica à base de leucita glazeada (IPS Empress) não houve alterações significante durante doze anos de escovação simulada na estabilidade de cor e rugosidade superficial, já a cerâmica de dissilicato de lítio (IPS e.max) obteve mudança na estabilidade de cor e rugosidade superficial após doze anos, mas sem resultados clinicamente considerável. Analisaram que o glazeamento atua como uma proteção ao efeito da escovação, um polimento adequado pode favorecer a resultados satisfatórios.

Yuan JCC, et al. (2017) avaliaram o efeito da escovação e a ciclagem térmica sobre a rugosidade superficial e estabilidade de cor em restaurações cerâmicas CAD/CAM. Em relação ao dissilicato de lítio não houve alteração significativa de cor, já a zircônia há maior diferença de cor após simulação clínica de 15 anos. Demonstra que a escovação torna as superfícies mais lisas do que a ciclagem térmica. Tanto para o dissilicato de lítio como para zircônia a mudança de cor foi abaixo do limite perceptível. As superfícies das cerâmicas ficaram com uma maior aspereza, porém, em relação à zircônia, se tornou suave após a escovação e a rugosidade superficial foi mínima.

Por outro lado, Koizumi $\mathrm{H}$, et al. (2015) avaliaram o brilho e a rugosidade de cerâmicas confeccionadas pelo método CAD/CAM. Para isso, os modelos foram submetidos à escovação durante 120 minutos, o que é equivalente a 5 anos. Esse estudo demonstrou que o brilho dos materiais foi reduzido após a escovação associado a uma maior rugosidade de superfície.

Além disso, Flury S, et al. (2017) estudaram sobre a rugosidade da superfície, a VITABLOCS Mark II apresenta rugosidade maior; a Lava Ultimate apresenta menor rugosidade; enquanto Paradigma MZ100, Vita Enamic e Ambarino alta-classe apresentaram rugosidades parecidas. Vale salientar que a rugosidade superficial foi influenciada pela utilização da técnica CAD/CAM, polimento e armazenamento em água. $O$ 
polimento demonstrou que diminui significantemente a rugosidade de superfície. Paradigma MZ100, Lava Ultimate e Ambarino classe alta apresentaram um melhor polimento, porém podem ser degradados quando expostos à escovação artificial e ao armazenamento dos materiais em água.

Por fim, Alencar-Silva FJ, et al. (2019) verificaram a influência de bebidas e escovação sobre a rugosidade superficial, cor e dureza de uma cerâmica de dissilicato de lítio CAD/CAM glazeada ou polida. Em suma, independente da bebida imersa a rugosidade superficial foi aumentada, em especial ao grupo glazeados, onde o chá preto causou maior diferença. A microdureza foi influenciada pelo tratamento de superficie, que por sua vez se mostrou diminuida, mesmo com essa diminuição os valores de dureza ainda se torna superior ao valor do esmalte dental.

\section{CONSIDERAÇÕES FINAIS}

Após uma síntese dos artigos utilizados para esta revisão de lietratura, o presente trabalho conclui que as cerâmicas de dissilicato de lítio CAD/CAM possuem propriedades satisfatórias em relação a sua resistência e estética, após exposição à fumaça do cigarro, associada ou não com a escovação. Vale ressaltar que é essencial um tratamento de superficie para reduzir a rugosidade da mesma, visto que com a rugosidade instalada, a escovação não é eficaz para altera-lá.

\section{REFERÊNCIAS}

1. ALANDIA-ROMAN CC, et al. Effect of cigarette smoke on color stability and surface roughness of dental composites. Journal of dentistry, 2013; 41: 73-79.

2. ALENCAR-SILVA FJ, et al. Effect of beverage solutions and toothbrushing on the surface roughness, microhardness, and color stainability of a vitreous CAD-CAM lithium disilicate ceramic. The Journal of prosthetic dentistry, 2019; 121: 711-716.

3. AL HAMAD KQ, et al. The Effect of Ceramic Type and Background Color on Shade Reproducibility of All-Ceramic Restorations. Journal of Prosthodontics, 2018; 29: 511-517.

4. ANADIOTI E, et al. Internal fit of pressed and computer-aided design/computer-aided manufacturing ceramic crowns made from digital and conventional impressions. The Journal of prosthetic dentistry, 2015; 113: 304-309.

5. AYAZ EA, et al. Effects of cigarette smoke and denture cleaners on the surface roughness and color stability of different denture teeth. Journal of Prosthetic Dentistry, 2014; 112: 241-248.

6. AZIZ A, et al. Clinical performance of chairside monolithic lithium disilicate glass-ceramic CAD-CAM crowns. Journal of Esthetic and Restorative Dentistry, 2019; 31: 613-619.

7. DARYAKENARI G, et al. Effect of Simulated Mastication on the Surface Roughness and Wear of Machinable Ceramics and Opposing Dental Enamel. Operative Dentistry, 2018; 44: 88-95.

8. DOS SANTOS DM, et al. Effect of different acidic solutions on the optical behavior of lithium disilicate ceramics. Journal of Prosthetic Dentistry, 2017; 118: 430-436.

9. EL GAMAL A, et al. Microhardness evaluations of CAD/CAM ceramics irradiated with $\mathrm{CO}_{2}$ or Nd:YAP laser. Laser therapy, 2017; $26: 13-18$.

10. FLURY S, et al. Effect of artificial toothbrushing and water storage on the surface roughness and micromechanical properties of tooth-colored CAD-CAM materials. Journal of Prosthetic Dentistry, 2017; 117: 767-774.

11. GARZA LA, et al. Effect of toothbrushing on shade and surface roughness of extrinsically stained pressable ceramics. Journal of Prosthetic Dentistry, 2016; 115: 489-494.

12. JAIN C, et al. Spectrophotometric evaluation of the color changes of different feldspathic porcelains after exposure to commonly consumed beverages. European Journal of Dentistry, 2013; 7: 80-172.

13. KOIZUMI H, et al. Surface roughness and gloss of current CAD/CAM resin composites before and after toothbrush abrasion. Dental Materials Journal, 2015; 34: 881-887.

14. LAWSON NC, BURGESS JO. Gloss and stain resistance of ceramic-polymer CAD/CAM restorative blocks. Journal of Esthetic and Restorative Dentistry, 2016; 28: 40-45.

15. MACIEL CL, et al. Influence of polishing systems on roughness and color change of two dental ceramics. J Adv Prosthodont, 2019; 11: 215-222.

16. MAHORSS ZH, et al. Effect of Cigarette Smoke on Surface Roughness of Different Denture Base Materials. Journal of Clinical and Diagnostic Research, 2015; 9: 39-42.

17. MENEZES SM, et al. Reabilitação estética do sorriso com laminados cerâmicos: Relato de caso clínico. Rev Odontol Bras Central, 2015; 24: 68-72.

18. MOTA GE, et al. The effect of milling and postmilling procedures on the surface roughness of CAD/CAM materials. J Esthet Restor Dent, 2017; 26: 450-458.

19. OZDOGAN A, DUYMUS ZY. Investigating the Effect of Different Surface Treatments on Vickers Hardness and Flexural Strength of Zirconium and Lithium Disilicate Ceramics. Journal of Prosthodontics, 2018; 29:129-135. 
20. YIN R, et al. Comparative evaluation of the mechanical properties of CAD/CAM dental blocks. Odontology, 2019; 107: 360-367.

21. SAMER MS, et al. Clinical Outcomes and Predictors of Satisfaction in Patients with Improved Lithium Disilicate AllCeramic Crowns. Medical Principles and Practice, 2017; 26: 470-479.

22. SCHESTATSKY R, et al. Microstructure, topography, surface roughness, fractal dimension, internal and marginal adaptation of pressed and milled lithium-disilicate monolithic restorations. Journal of Prosthodontic Research, 2020; 64: 12-19.

23. STRASSER T, et al. Roughness, surface energy, and superficial damages of CAD/CAM materials after surface treatment. Clinical oral investigations, 2018; 22: 2787-2797.

24. SUDRÉ JP, et al. Influence of Surface Treatment of Lithium Disilicate on Roughness and Bond Strength. The International Journal of Prosthodontics, 2020; 33: 212-216.

25. TSAI MM, et al. Effect of cigarette smoking on the bond strength between resin cement and dental CAD/CAM ceramics. Journal of Adhesion Science and Technology, 2018; 31: 2323-2334, 2018.

26. VICHI A, et al. Effect of Finishing and Polishing on Roughness and Gloss of Lithium Disilicate and Lithium Silicate Zirconia Reinforced Glass Ceramic for CAD/CAM Systems. Operative Dentistry,2018; 43: 90-100.

27. YUAN JCC, et al. Effect of brushing and thermocycling on the shade and surface roughness of CAD-CAM ceramic restorations. The Journal of prosthetic dentistry, 2018; 119: 1000-1006.

28. ZELTNER M, et al. Randomized controlled within-subject evaluation of digital and conventional workflows for the fabrication of lithium disilicate single crowns. Part III: marginal and internal fit. J Prosthet Dent, 2017; 117: $354-362$. 